\title{
A COVERING LEMMA FOR PRODUCT SPACES
}

\author{
JEAN-LIN JOURNÉ \\ Dedicated to Mischa Cotlar
}

\begin{abstract}
We give a substitute for the Whitney decomposition of an arbitrary open set in $\mathbf{R}^{2}$ where squares are replaced by rectangles. Then we deduce the $L^{\infty}$-BMO boundedness of certain singular integral operators defined on product spaces.
\end{abstract}

In [2] it is shown that $\mathrm{BMO}(\mathbf{R} \times \mathbf{R})$, the dual of $H^{\mathbf{1}}(\mathbf{R} \times \mathbf{R})$, can be characterized in terms of Carleson measures on $\mathbf{R}_{+}^{2} \times \mathbf{R}_{+}^{2}[\mathbf{1}]$. Let $\Omega$ be a bounded open set in $\mathbf{R}^{2}$ and $S(\Omega)$ be the shadow region over $\Omega$, that is, the set of $\left(x_{1}, x_{2}, t_{1}, t_{2}\right)$ such that $\left[x_{1}-t_{1}, x_{1}+t_{1}\right] \times\left[x_{2}-t_{2}, x_{2}+t_{2}\right] \subseteq \Omega$. Let $\psi \in C_{0}^{\infty}(\mathbf{R})$ be supported on $[-1,1]$, such that $\int \psi d x=0$, and let $Q_{t}=Q_{t_{1}} \otimes Q_{t_{2}}$ be the convolution with $\left(1 / t_{1} t_{2}\right) \psi\left(x_{1} / t_{1}\right) \psi\left(x_{2} / t_{2}\right)$. Then a function $b \in L_{\text {loc }}^{2}\left(\mathbf{R}^{2}\right)$ is in $\operatorname{BMO}(\mathbf{R} \times \mathbf{R})$ if and only if there exists a constant $C_{b}$ such that

$$
\int_{S(\Omega)}\left|Q_{t} b\right|^{2} d x_{1} d x_{2} \frac{d t_{1}}{t_{1}} \frac{d t_{2}}{t_{2}} \leq C_{b}^{2}|\Omega|
$$

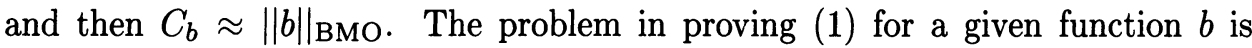
that one has to consider all possible bounded open sets and not simply rectangles. However, (1) can still be checked in special cases, using the following proposition.

PROPOSITION 1. Let $\Omega$ be a bounded open subset of $\mathbf{R}^{2}$, let $\left\{R_{k}, k \in K\right\}$ be the collection of maximal dyadic rectangles contained in $\Omega$, and let $R_{k}=I_{k} \times J_{k}$ for all $k \in K$. There exist dyadic intervals $\left\{\tilde{I}_{k}, k \in K\right\}$ such that $I_{k} \subseteq \tilde{I}_{k}$ for all $k \in K$ and such that for all increasing functions $\omega:\left\{2^{-j}, j \in N\right\} \rightarrow[0,+\infty)$

$$
\left\|\bigcup_{k \in K} \tilde{I}_{k} \times J_{k}\right\| \leq 2|\Omega|
$$

and

$$
\sum_{k \in K}\left|R_{k}\right| \omega\left(\frac{\left|I_{k}\right|}{\left|\tilde{I}_{k}\right|}\right) \leq 2\left[\sum_{j=0}^{+\infty} \omega\left(2^{-j}\right)\right]|\Omega| .
$$

An alternate way to state this proposition in nondyadic language is the following. For all $\left(x_{1}, t_{1}\right) \in R_{+}^{2}$ let $E_{x_{1} t_{1}}$ be the open set $\left\{x_{2},\left[x_{1}-t_{1}, x_{1}+t_{1}\right] \times\left\{x_{2}\right\} \subseteq \Omega\right\}$ and $\bigcup_{l} I_{x_{1} t_{1}}^{l}$ be its decomposition in connected components.

Received by the editors November 27, 1984.

1980 Mathematics Subject Classification. Primary 42-00.

Key words and phrases. Carleson measures, Whitney decompostion.

(C)1986 American Mathematical Society $0002-9939 / 86 \$ 1.00+\$ .25$ per page 
PROPOSITION 2. There exists a function $t_{1}^{l}\left(x_{1}, t_{1}\right)$ such that

$$
\left.\| \bigcup_{x_{1}, t_{1}, l}\right] x_{1}-t_{1}^{l}, x_{1}+t_{1}^{l}\left[\times I_{x_{1} t_{1}}^{l} \| \leq 8|\Omega|\right.
$$

and such that, for all $\omega:[0,1] \rightarrow[0,+\infty)$ increasing,

$$
\int_{x_{1}} \int_{t_{1}} \sum_{l}\left|I_{x_{1} t_{1}}^{l}\right| \omega\left(\frac{t_{1}}{t_{1}^{l}}\right) \frac{d x_{1} d t_{1}}{t_{1}} \leq 2\left[\int_{0}^{1} \frac{\omega(\delta)}{\delta} d \delta\right]|\Omega| .
$$

Propositions 1 and 2 are formally the same and so are their proofs. Thus we shall only prove Proposition 2.

The function $t_{1}^{l}$ is simply defined as

$$
\inf \left\{t_{1}^{\prime} \geq t_{1},\left|E_{x_{1} t_{1}^{\prime}} \cap I_{x_{1} t_{1}}^{l}\right| /\left|I_{x_{1} t_{1}}^{l}\right| \leq 1 / 2\right\} .
$$

To show (4) we observe that if $t_{1}^{\prime}<t_{1}^{l}$, then $\left|E_{x_{1} t_{1}^{\prime}} \cap I_{x_{1} t_{1}}^{l}\right|>\frac{1}{2}\left|I_{x_{1} t_{1}}^{l}\right|$, and, therefore, since $] x_{1}-t_{1}^{\prime}, x_{1}+t_{1}^{\prime}\left[\times E_{x_{1} t_{1}^{\prime}} \subseteq \Omega\right.$,

$$
\mid] x_{1}-t_{1}^{\prime}, x_{1}+t_{1}^{\prime}\left[\times I_{x_{1} t_{1}}^{l} \cap \Omega\left|>\frac{1}{2} \times 2 t_{1}^{\prime} \times\right| I_{x_{1} t_{1}}^{l} \mid .\right.
$$

If we let $\Omega_{1}$ be $\left.\bigcup_{x_{1}, t_{1}, l}\right] x_{1}-t_{1}^{l}, x_{1}+t_{1}^{l}\left[\times I_{x_{1} t_{1}}^{l}\right.$ and if $*$ denotes the strong HardyLittlewood maximal function then $\frac{1}{2} \chi_{\Omega_{1}} \leq \chi_{\Omega}^{*}$, which implies (4).

To estimate the l.h.s of (5) we rewrite it as

$$
\int_{x_{1}} \int_{t_{1}} \sum_{l} \int_{t_{1}^{\prime}>t_{1}^{l}}\left|I_{x_{1} t_{1}}^{l}\right| \omega^{\prime}\left(\frac{t_{1}}{t_{1}^{\prime}}\right) \frac{d t_{1}^{\prime}}{t_{1}^{\prime 2}} d t_{1} d x_{1}
$$

assuming that $\omega$ is in $C^{1}[0,1]$, which is no loss in generality. If $t_{1}^{\prime}>t_{1}^{l}$, then $\left|E_{x_{1} t_{1}^{\prime}} \cap I_{x_{1} t_{1}}^{l}\right| \leq \frac{1}{2}\left|I_{x_{1} t_{1}}^{l}\right|$, so that $\left|I_{x_{1} t_{1}}^{l} \backslash E_{x_{1} t_{1}^{\prime}}\right| \geq \frac{1}{2}\left|I_{x_{1} t_{1}}^{l}\right|$. Hence, (6) is less than

$$
\int_{x_{1}} \int_{t_{1}} \sum_{l} \int_{t_{1}^{\prime}>t_{1}^{l}}\left[\int_{I_{x_{1} t_{1}}^{l} \backslash E_{x_{1} t_{1}^{\prime}}} 2 d x_{2}\right] \omega^{\prime}\left(\frac{t_{1}}{t_{1}^{\prime}}\right) \frac{d t_{1}^{\prime}}{t_{1}^{\prime 2}} d t_{1} d x_{1} .
$$

For $\left(x_{1}, x_{2}\right) \in \Omega$ fixed, let

$$
T_{1}\left(x_{1}, x_{2}\right)=\sup \left\{s_{1},\left[x_{1}-s_{1}, x_{1}+s_{1}\right] \times\left\{x_{2}\right\} \subseteq \Omega\right\} .
$$

If $x_{2} \in I_{x_{1} t_{1}}^{l} \backslash E_{x_{1} t_{1}^{\prime}}$, then $t_{1} \leq T_{1}\left(x_{1}, x_{2}\right) \leq t_{1}^{\prime}$. Hence, (7) is less than

$$
2 \iint_{\left(x_{1}, x_{2}\right) \in \Omega}\left[\int_{t_{1} \leq T_{1} \leq t_{1}^{\prime}} \omega^{\prime}\left(\frac{t_{1}}{t_{1}^{\prime}}\right) \frac{d t_{1}^{\prime}}{t_{1}^{\prime 2}}\right] d x_{1} d x_{2}
$$

which is exactly $2\left[\int_{0}^{1} \omega(\delta) \frac{d \delta}{\delta}\right] \times|\Omega|$.

As we said, Proposition 1 is nothing but Proposition 2 reformulated in dyadic language. It also has the advantage of extending immediately to a product of type $\mathbf{R}^{n} \times \mathbf{R}^{m}$. For simplicity we stick to the case of $\mathbf{R} \times \mathbf{R}$ and consider the following problem. Let $T_{1}$ and $T_{2}$ be two Calderon-Zygmund operators as defined in [3] or [5]. Then $T_{1} \otimes T_{2}$ is trivially bounded on $L^{2}\left(\mathbf{R}^{2}\right)$ and on $L^{p}\left(\mathbf{R}^{2}\right)$ for all $\left.p \in\right] 1,+\infty[$. Moreover, we have the following. 
PROPOSITION 3. Let $T_{1}$ and $T_{2}$ be two Calderon-Zygmund operators acting on $L^{2}(\mathbf{R})$. Then $T_{1} \otimes T_{2}$ extends to an operator bounded from $L^{\infty}\left(\mathbf{R}^{2}\right)$ to $\mathrm{BMO}(\mathbf{R} \times \mathbf{R})$.

As is readily seen, the proof we give below requires only a very weak smoothness assumption on the kernels of $T_{1}$ and $T_{2}$. Let $T$ be a Calderon-Zygmund operator acting on $L^{2}(\mathbf{R})$. Then $Q_{t} T$ is well defined for all $t>0$ and has a kernel $\left(Q_{t} T\right)(x, y)$, given when $|x-y|>t$ by

$$
\int \psi_{t}(x-u)[K(u, y)-K(x, y)] d u
$$

where $K$ is the kernel of $T$. What we shall need is that for all $x \in \mathbf{R}$ and $t>0$,

$$
\int_{t_{0}<|x-y| / 2}\left|\left(Q_{t_{0}} T\right)(x, y)\right| d y<C(T)
$$

and

$$
\begin{aligned}
\iint_{t<t_{0}<|x-y| / 2}\left|\left(Q_{t} T\right)(x, y)\right| d y<\omega_{T}\left(\frac{t}{t_{0}}\right) \\
\quad \text { with } \int_{0}^{1} \omega_{T}(\delta) \frac{d \delta}{\delta}=C(T)<+\infty .
\end{aligned}
$$

This will certainly be the case if the function $\omega$, defined as

$$
\omega(\delta)=\sup _{x, x^{\prime}} \int_{|x-y|>2\left|x-x^{\prime}\right| / \delta}\left|K(x, y)-K\left(x^{\prime}, y\right)\right| d y,
$$

satisfies $\omega(1)<+\infty$ and the Dini condition $\int_{0}^{1} \omega(\delta) / \delta d \delta<+\infty$.

We should also remark that this proof extends with minor changes to the class of singular integral operators defined in [4] or [6].

To prove Proposition 3 we choose a bounded open set $\Omega$ in $\mathbf{R}^{2}$, a function $b \in C_{0}^{\infty}\left(\mathbf{R}^{2}\right)$ such that $\|b\|_{\infty}<1$, two Calderon-Zygmund operators $T_{1}$ and $T_{2}$ satisfying $\left(8^{\prime}\right)$ and $\left(8^{\prime \prime}\right)$ with a constant 1 and such that $\left\|T_{1}\right\|_{2,2}+\left\|T_{2}\right\|_{2,2} \leq 1$. Then we must show that

$$
\int_{S(\Omega)}\left|Q_{t_{1}} Q_{t_{2}} S b\right|^{2} d x_{1} d x_{2} \frac{d t_{1}}{t_{1}} \frac{d t_{2}}{t_{2}} \leq C|\Omega|
$$

where $S$ denotes $T_{1} \otimes T_{2}$.

Recall that, by Plancherel's theorem, if $f \in L^{2}\left(\mathbf{R}^{2}\right)$,

$$
\iint_{\mathbf{R}_{+}^{2} \times \mathbf{R}_{+}^{2}}\left|Q_{t_{1}} Q_{t_{2}} f\right|^{2} d x_{1} d x_{2} \frac{d t_{1}}{t_{1}} \frac{d t_{2}}{t_{2}} \leq C\|f\|_{2}^{2}
$$

Applying this to $f=S\left(b \chi_{\Omega_{2}}\right)$, where $\Omega_{2}=\bigcup_{R \subseteq \Omega_{1}} 2 R$, and using

$$
\|f\|_{2}^{2} \leq\|S\|_{2,2}^{2}\|b\|_{\infty}^{2}\left|\Omega_{2}\right| \leq C|\Omega|,
$$

we see that we may as well suppose that $b$ is supported outside of $\Omega_{2}$.

For each $\left(x_{2}, t_{2}\right) \in \mathbf{R}_{+}^{2}$ let $\left.F_{x_{2} t_{2}}=\bigcup \int x_{1}-2 t_{1}^{l}, x_{1}+2 t_{1}^{l}\right]$, where the union is over those $\left(x_{1}, t_{1}, l\right)$ such that $\left[x_{2}-t_{2}, x_{2}+t_{2}\right] \subseteq I_{x_{1} t_{1}}^{l}$. Observe that

$$
\left.\bigcup_{x_{2}, t_{2}} F_{x_{2} t_{2}} \times\right] x_{2}-2 t_{2}, x_{2}+2 t_{2}\left[\subseteq \Omega_{2} .\right.
$$


We now split $b$ as $b_{x_{2} t_{2}}^{1}+b_{x_{2} t_{2}}^{2}$, as in [1], but in a different way. Here $b_{x_{2} t_{2}}^{1}\left(z_{1}, z_{2}\right)=$ $b\left(z_{1}, z_{2}\right) \chi_{F_{x_{2} t_{2}}}\left(z_{1}\right)$, so that $b_{x_{2} t_{2}}^{1}\left(z_{1}, z_{2}\right)=0$ for all $z_{1}$ if $\left|z_{2}-x_{2}\right| \leq 2 t_{2}$, since $b$ is supported out of $\Omega_{2}$.

In order to prove (9) with $b_{x_{t} t_{2}}^{1}$ instead of $b$, observe that $\left[Q_{t_{1}} Q_{t_{2}} S b_{x_{2} t_{2}}^{1}\right]\left(x_{1}, x_{2}\right)$ can be written as

$$
\int_{\left|x_{2}-z_{2}\right| \geq 2 t_{2}}\left(Q_{t_{2}} T_{2}\right)\left(x_{2}, z_{2}\right)\left[Q_{t_{1}} T_{1} b_{x_{2} t_{2}}^{1}\left(\cdot, z_{2}\right)\right]\left(x_{1}\right) d z_{2}
$$

Minkowski's inequality implies that

$$
\iint\left|\left(Q_{t_{1}} Q_{t_{2}} S b_{x_{2} t_{2}}^{1}\right)\left(x_{1}, x_{2}\right)\right|^{2} \frac{d x_{1} d t_{1}}{t_{1}}
$$

is less than

$$
\left[\int_{\left|x_{2}-z_{2}\right| \geq 2 t_{2}}\left|\left(Q_{t_{2}} T_{2}\right)\left(x_{2}, z_{2}\right)\right|\left[\iint\left|\left[Q_{t_{1}} T_{1} b_{x_{2} t_{2}}^{1}\left(\cdot, z_{2}\right)\right]\left(x_{1}\right)\right|^{2} \frac{d x_{1} d t_{1}}{t_{1}}\right]^{1 / 2} d z_{2}\right]^{2} .
$$

Using Cauchy-Schwarz, $\left(8^{\prime}\right)$ and the $L^{2}$-boundedness of $T_{1}$, we see that this integral is dominated by

$$
\iint_{\left(z_{1}, z_{2}\right) \in \operatorname{supp}}\left|\left(Q_{t_{2}} T_{2}\right)\left(x_{2}, z_{2}\right)\right| d z_{1} d z_{2}
$$

We integrate (10) with respect to $d x_{2} d t_{2} / t_{2}$ and fix $x_{2}$ and $z_{1}$. Observe that $\left(z_{1}, x_{2}\right) \in \Omega_{2}$ if $z_{1} \in F_{x_{2} t_{2}}$ for some $t_{2} \geq 0$. Therefore, to prove (9) with $b_{x_{2} t_{2}}^{1}$ instead of $b$ it is enough to prove

$$
\iint_{\left(z_{2}, t_{2}\right) \in A_{x_{2} z_{1}}}\left|\left(Q_{t_{2}} T_{2}\right)\left(x_{2}, z_{2}\right)\right| d z_{2} \frac{d t_{2}}{t_{2}} \leq C
$$

where

$$
A_{x_{2} z_{1}}=\left\{\left(z_{2}, t_{2}\right),\left|x_{2}-z_{2}\right| \geq 2 t_{2},\left(z_{1}, z_{2}\right) \in \operatorname{supp} b_{x_{2} t_{2}}^{1}\right\} .
$$

Let $\tau\left(z_{1}, x_{2}\right)=\sup \left\{t_{2}, z_{1} \in F_{x_{2} t_{2}}\right\}$. If $\left(z_{1}, z_{2}\right) \in \operatorname{supp} b_{x_{2} t_{2}}^{1}$, then $t_{2} \leq \tau\left(z_{1}, x_{2}\right)$. On the other hand,

$$
\left.\left\{z_{1}\right\} \times\right] x_{2}-2 \tau, x_{2}+2 \tau\left[\subseteq \bigcup_{t_{2}} F_{x_{2} t_{2}} \times\right] x_{2}-2 t_{2}, x_{2}+2 t_{2}\left[\subseteq \Omega_{2} .\right.
$$

Therefore if $\left(z_{1}, z_{2}\right) \in \operatorname{supp} b_{x_{2} t_{2}}^{1},\left(z_{1}, z_{2}\right) \in \Omega_{2}^{c}$ and $\left|x_{2}-z_{2}\right| \geq 2 \tau$, so that if $\left(z_{2}, t_{2}\right) \in A_{x_{2} z_{1}}$, then $t_{2} \leq \tau \leq\left|x_{2}-z_{2}\right| / 2$. This, together with ( $\left.8^{\prime \prime}\right)$, implies (11).

The proof of (9) with $b_{x_{2} t_{2}}^{2}$ instead of $b$ is along the same lines. As in [1] we rewrite the l.h.s. of $(9)$ as

$$
\iint_{\left(x_{1}, t_{1}\right)}\left(\sum_{l}\left[\int_{S\left(I_{x_{1} t_{1}}^{l}\right)}\left|Q_{t_{1}} Q_{t_{2}} S b_{x_{2} t_{2}}^{2}\left(x_{1}, x_{2}\right)\right|^{2} \frac{d x_{2} d t_{2}}{t_{2}}\right]\right) \frac{d x_{1} d t_{1}}{t_{1}}
$$

Recall that $b_{x_{2} t_{2}}^{2}\left(z_{1}, z_{2}\right)=b \chi_{F_{x_{2} t_{2}}^{c}}\left(z_{1}\right)$. If $\left(x_{2}, t_{2}\right) \in S\left(I_{x_{1} t_{1}}^{l}\right)$ and $z_{1} \notin F_{x_{2} t_{2}}$, then, by definition, $\left|x_{1}-z_{1}\right| \geq 2 t_{1}^{l}\left(x_{1}, t_{1}\right)$. Therefore, $b_{x_{2} t_{2}}^{2}\left(z_{1}, z_{2}\right)=0$ if $\left|z_{1}-x_{1}\right| \leq$ $2 t_{1}^{l}$. Now we develop in the variable $z_{1}$ as we did before in the variable $z_{2}$, use 
Minkowski's inequality in $z_{1}$, the $L^{\infty}$-BMO boundedness of $T_{2}$, and finally we are reduced to estimating

$$
\iint_{\left(x_{1} t_{1}\right)}\left[\sum_{l} \int_{\left|x_{1}-z_{1}\right| \geq 2 t_{1}^{l}}\left|I_{x_{1} t_{1}}^{l}\right|\left|\left(Q_{t_{1}} T\right)_{x_{1} z_{1}}\right| d z_{1}\right] \frac{d x_{1} d t_{1}}{t_{1}}
$$

By $\left(8^{\prime \prime}\right)$ and (5) this is less than $C|\Omega|$. Proposition 3 is proved.

Recall that in one variable one associates to any function $\psi \in C_{0}^{\infty}(R)$ with $\int \psi d x=0$ a Littlewood-Paley $G$-function defined by

$$
G f(x)=\left[\int_{0}^{+\infty}\left(\psi_{t} * f\right)^{2}(x) \frac{d t}{t}\right]^{1 / 2}
$$

By Plancherel's theorem $\|G f\|_{2} \leq C\|f\|_{2}$. Using vector-valued singular integral operators, we see also that $\|G f\|_{\text {BMO }} \leq C\|f\|_{\text {BMO }}$. It is known that $\left\|(G f)^{2}\right\|_{\text {BMO }} \leq$ $C\|f\|_{\mathrm{BMO}}^{2}$. We shall generalize this latter fact to the product situation.

Proposition 4. Let $\psi \in C_{0}^{\infty}(R)$ with $\int \psi d x=0$, and let $Q_{t}$ be the convolution with $(1 / t) \psi(\cdot / t)$. For all $f \in L_{\text {loc }}^{2}\left(\mathbf{R}^{2}\right)$, let

Then $\left\|(G f)^{2}\right\|_{\mathrm{BMO}(\mathbf{R} \times \mathbf{R})} \leq C\|f\|_{\mathrm{BMO}(\mathbf{R} \times \mathbf{R})}^{2}$.

$$
G f(x)=\left[\int_{0}^{+\infty} \int_{0}^{+\infty}\left[\left(Q_{t_{1}} Q_{t_{2}} f\right)^{2}\left(x_{1}, x_{2}\right)\right]^{2} \frac{d t_{1} d t_{2}}{t_{1} t_{2}}\right]^{1 / 2}
$$

To prove this proposition it is enough to show that the operator $T_{f}$ defined for $f \in C_{0}^{\infty}\left(\mathbf{R}^{2}\right)$ by

$$
T_{f}(g)=\int_{0}^{+\infty} \int_{0}^{+\infty}\left(Q_{t_{1}} Q_{t_{2}} f\right)\left(Q_{t_{1}} Q_{t_{2}} g\right) \frac{d t_{1}}{t_{1}} \frac{d t_{2}}{t_{2}}
$$

for $g$ in $C_{0}^{\infty}\left(\mathbf{R}^{2}\right)$ extends to an operator bounded from $\mathrm{BMO}(\mathbf{R} \times \mathbf{R})$ to itself with a norm at most $C\|f\|_{\text {BMO }}$. Actually it is rather easy to see that $T_{f}$ is a CZO on $\mathbf{R} \times \mathbf{R}$ as defined in [6], to which the T1-theorem of [6] applies and also Proposition 3. Knowing that $T_{f}$ maps $L^{\infty}$ to BMO, it remains to show that $T_{f} H_{1}, T_{f} H_{2}, T_{f} H_{1} H_{2}$, where $H_{1}$ and $H_{2}$ are the partial Hilbert transforms, also map $L^{\infty}$ to BMO. Then the decomposition of BMO functions in terms of $L^{\infty}$ and partial Hilbert transforms will imply that $T_{f}$ maps BMO to itself. Now the fact that $T_{f} H_{1}, T_{f} H_{2}, T_{f} H_{1} H_{2}$ are CZOs to which Proposition 3 applies follows easily from the fact that the kernel of $Q_{t} H$ has the smoothness and decay properties of the Poisson kernel $p_{t}$.

Another approach to the $L^{2}$-boundedness of $T_{f}$ uses the technique of [3, Chapter VI]. This is more powerful since one can also prove vector-valued inequalities. Let $V$ be the Hilbert space

$$
L^{2}\left(\left(\mathbf{R}_{+}\right)^{2}, \frac{d t_{1}}{t_{1}} \frac{d t_{2}}{t_{2}}\right)
$$

Let $g$ be a $V$-valued $C_{0}^{\infty}\left(\mathbf{R}^{2}\right)$ function. Then $\left[\tilde{T}_{f}(g)\right](x)$ is defined as

$$
\int_{0}^{+\infty} \int_{0}^{+\infty}\left[\left(Q_{t_{1}} Q_{t_{2}} f\right)(x)\right]\left[\left(Q_{t_{1}} Q_{t_{2}} g\left(\cdot, t_{1}, t_{2}\right)\right)(x)\right] \frac{d t_{1}}{t_{1}} \frac{d t_{2}}{t_{2}}
$$

Then $\tilde{T}_{f}$ is also bounded from $L_{V}^{2}\left(\mathbf{R}^{2}\right)$ to $L^{2}\left(\mathbf{R}^{2}\right)$ and from $\mathrm{BMO}_{V}(\mathbf{R} \times \mathbf{R})$ to $\operatorname{BMO}(\mathbf{R} \times \mathbf{R})$.

ACKNOWLEDGment. I would like to thank Guy David, Stephen Semmes and Fernando Soria for reading this manuscript and suggesting numerous improvements. 


\section{REFERENCES}

1. S.-Y. A. Chang, Carleson measure on the bi-disc, Ann. of Math. (2) 109 (1979), 613-620.

2. S.-Y. A. Chang and R. Fefferman, A continuous version of duality of $H^{1}$ with BMO on the bi-disc, Ann. of Math. (2) 112 (1980), 179-201.

3. R. R. Coifman and Y. Meyer, Au delà des opérateurs pseudo-differentiels, Astérisque No. $57,1978$.

4. R. Fefferman and E. M. Stein, Singular integrals on product spaces, Adv. in Math. 45 (1982), 117-143.

5. J.-L. Journé, Calderón-Zygmund operators, pseudo-differential operators and the Cauchy integral of Calderón, Lecture Notes in Math., vol. 994, Springer-Verlag, Berlin and New York, 1983.

6. __ Calderón-Zygmund operators on product spaces, Revista Ibero-Americana 3 (1985).

Department of Mathematics, Yale University, New haven, Connecticut 06520

Current address: Department of Mathematics, Princeton University, Princeton, New Jersey 08544 